\title{
PISCICULTURA MARINHA BRASILEIRA: DESAFIOS E PERSPECTIVAS DO SEU DESENVOLVIMENTO NO ESTADO DO CEARÁ
}

\author{
Viviana Lisboa \\ viviana.lisboa.lisboa@gmail.com \\ Fundação Cearense de Apoio \\ ao Desenvolvimento Científico \\ e Tecnológico, Secretaria do \\ Desenvolvimento Agrário, \\ Fortaleza, Ceará, Brasil
}

Halana Rodrigues Freire Eloy halanarodrigues@gmail.com Fundação Cearense de Apoio ao Desenvolvimento Científico e Tecnológico, Secretaria do Desenvolvimento Agrário, Fortaleza, Ceará, Brasil

\section{Karla Maria Catter}

kmcatter@yahoo.com.br Fundação Cearense de Apoio ao Desenvolvimento Científico e Tecnológico, Secretaria do Desenvolvimento Agrário, Fortaleza, Ceará, Brasil

\section{Rochelle Cruz Araújo Bezerra} Vidigal

rochelle.cruz@cedepesca.net Fundação Cearense de Apoio ao Desenvolvimento Científico e Tecnológico, Secretaria do Desenvolvimento Agrário, Fortaleza, Ceará, Brasil

\section{Rossi Lelis Muniz Souza}

rossilelis@gmail.com

Fundação Cearense de Apoio ao Desenvolvimento Científico e Tecnológico, Secretaria do Desenvolvimento Agrário, Fortaleza, Ceará, Brasil

João Felipe Nogueira Matias

jfn.matias@gmail.com

Fundação Cearense de Apoio

ao Desenvolvimento Científico

e Tecnológico, Secretaria do

Desenvolvimento Agrário,

Fortaleza, Ceará, Brasil

\section{RESUMO}

A pesca ainda é a maior responsável pelo fornecimento de pescado marinho para consumo humano. Entretanto, visto a notória ameaça aos estoques pesqueiros e a crescente demanda pelo produto, este cenário precisa mudar e a piscicultura marinha continua sendo mundialmente apontada como medida viável para remediar esse quadro. Desta forma, o objetivo deste artigo é discutir sobre a piscicultura marinha no Brasil, com foco nos desafios e perspectiva de seu desenvolvimento no estado do Ceará. Durante o período de seis meses, a metodologia do trabalho constituiu-se de três ferramentas: levantamento bibliográfico, entrevistas e visitas técnicas. Os resultados obtidos retratam que, apesar de todas as inciativas públicas e privadas, a piscicultura marinha no Brasil continua incipiente e com obstáculos a serem superados. No que concerne ao Ceará, o estado, por intermédio de órgão de fomento, tem executado ações com o propósito de oportunizar o desenvolvimento da piscicultura marinha. Dentre as ações planejadas, incialmente está sendo executado a elaboração de projeto de laboratório para provimento de alevinos de espécies marinhas, simultaneamente a sua análise da viabilidade econômica financeira, e a prospecção de espécies profícuas para produção no estado, cuja conjectura preliminar tem apontado para Lutjanus synagris (ariacó) e, sobretudo, Lutjanus analis (cioba).

Palavras-chave: Aquicultura; Maricultura; Piscicultura; Marinha. 


\section{INTRODUÇÃO}

Segundo a legislação brasileira, aquicultura é a atividade de cultivo de organismos cujo ciclo de vida em condições naturais se dá total, ou parcialmente, em meio aquático, implicando a propriedade do estoque sob cultivo, equiparada à atividade agropecuária (Brasil, 2009). Dentre as categorias da aquicultura temos a maricultura, que consiste na produção de organismos aquáticos especificamente em água salgada ou salobra, destacando-se, de modo geral, a algicultura marinha (cultivos de algas), a equinodermocultura (cultivo de equinodermos), a malacocultura marinha (cultivo de moluscos), a carcinicultura marinha (cultivos de crustáceos) e a piscicultura marinha (cultivo de peixes marinhos).

Da produção mundial de pescado em 2018, correspondente a 178,5 milhões de toneladas, 96,4 milhões foram fornecidas pela pesca e 82,1 milhões pela aquicultura. Destaca-se que das 82,1 milhões de toneladas oriundas da aquicultura, 30,8 milhões foram produzidas pela maricultura, sendo 7,3 milhões toneladas especificamente da produção de peixes marinhos (FAO, 2020).

Apesar dos 84,4 milhões de toneladas de pescados produzidos pela pesca, especificamente marinha, é notório que os estoques pesqueiros de interesse comercial já se encontraram ameaçados de sobre-explotação, ou sobre-explotados, e que, na contramão da preservação, a demanda por este tipo de produto é crescente (FAO, 2020). Essa problemática reforça a necessidade da implementação de alternativas eficazes e sustentáveis para prover o fornecimento dessa fonte de proteína de alto valor biológico, que é o pescado (Camargo; Pouey, 2005; Gonçalves, 2011). Visto que a piscicultura marinha é mundialmente apontada para esta finalidade, este artigo busca realizar uma discussão sobre a piscicultura marinha no Brasil, com foco nos desafios e na perspectiva para o seu desenvolvimento no estado do Ceará.

\section{METODOLOGIA}

Para essa proposta, a metodologia utilizada constituiu-se de três técnicas de coleta de dados, realizadas em um período de seis meses, sendo gastos dois meses em cada etapa: levantamento bibliográfico, entrevistas e visitas técnicas.

A revisão bibliográfica foi realizada em revistas científicas detentoras de Qualis Capes, revistas técnicas especializadas e de destaque na área de aquicultura e portais eletrônicos oficiais de empresas (i.e. Embrapa, Epagri) que desenvolvem ações na área de Maricultura. Para realização do levantamento bibliográfico foram utilizados termos como "Maricultura", "Piscicultura Marinha" e "Produção de Peixes Marinhos". No decorrer da revisão bibliográfica também foram utilizados termos relacionados aos nomes científicos e comuns das es- pécies de peixes marinhos (i.e. bijupirá, Rachycentron, vermelhos, Lutjanídeos) apontadas para produção em cativeiro, nos tópicos abordados (i.e. reprodução, larvicultura e nutrição), nas principais ações públicas e privadas, realizadas e em execução, e no status atual da atividade. As entrevistas foram realizadas com os cinco principais pesquisadores que exercem e exerceram papéis ativos no cenário da piscicultura marinha brasileira e cearense, com os dois atuais, e únicos, produtores comerciais de engorda de peixe marinho, com a atual, e única, empresa comercial fornecedora de alevinos de peixes marinhos, com um ex-produtor de engorda de peixe marinho, com o ex-fornecedor principal de insumos para produção de alimento vivo e larvicultura de peixes marinhos e com os representantes comerciais das duas atuais empresas fornecedoras de ração para peixes marinho. Os entrevistados (pesquisadores, produtores, ex-produtores e representantes comerciais) foram questionados sobre o seu histórico de atuação no cenário da piscicultura marinha, quais os principais entraves enfrentados e perspectivas, ou não, para o setor. As visitas técnicas por sua vez foram direcionadas a quatro laboratórios referência em pesquisa sobre piscicultura marinha, sendo dois ativos no Brasil (Rio Grande do Sul e Santa Catarina), um no Ceará (atualmente inoperante especificamente na linha de piscicultura marinha) e um na Colômbia.

\section{RESULTADOS}

\section{Panorama da piscicultura marinha no Brasil}

Mundialmente, a origem da piscicultura marinha é desconhecida. A obra Clássico de Cultivo de Peixes, considerada o primeiro registro, parece ter sido escrita no ano 500 a.C. por um político chinês chamado Fan Lei (Pillay, 1993). Já o desenvolvimento da piscicultura marinha em escala comercial ocorreu no Japão, na década de 1960, com a descoberta de que o rotífero (um tipo de zooplâncton) poderia ser utilizado como alimento vivo para alimentação de larvas de peixes marinhos (Hirata, 1979; Cerqueira, 2004; Côrtes; Tsuzuki, 2010). Atualmente, os dados mais recentes apontam para a produção mundial de 7,3 milhões de toneladas de peixes marinhos, sendo o salmão (Salmo salar) a principal espécie, e a Noruega e o Chile os principais produtores de salmão (FAO, 2020).

No Brasil, a criação de peixes marinhos teve início no estado de Pernambuco, no século XVII, quando da ocupação dos holandeses, através da capturada e confinamento em viveiros. Neste período, as principais espécies mantidas em sistema extensivo de viveiros de maré nos municípios de Recife e Olinda foram robalos (Centropomus), tainhas (Mugil) e carapebas (Eugerres e Diapterus) (Von Ihering, 1932; Cavalli; Hamilton, 2009). Na década de 1930, Schubart (1936) 
estimou que havia uma produção anual de 25 toneladas em uma área de 43 hectares de viveiro na região de Recife.

De forma geral, os critérios utilizados para seleção de espécies de peixes marinhos com potencial para produção em cativeiro são: possuir preço de mercado, efetuar reprodução em cativeiro (para fornecimento de alevinos), adaptar-se ao cativeiro e ao consumo de dieta artificial (ração), apresentar potencial de crescimento em cativeiro, ser resistente (espécie rústica) e de fácil manejo (Sampaio et al., 2001; Tutman et al., 2004; Cavalli; Hamilton 2007; Cunha et al., 2013; Cerqueira et al., 2017).

Baseado nesses critérios, tainhas ( $M$. liza), linguados (Paralichthys orbignyanus), robalos (Centropomus parallelus a C. undecimalis), bijupirás (Rachycentron canadum), peixes vermelhos (Lutjanus spp.), garoupas (Epinephelus marginatus) e, mais recentemente, sardinhas (Sardinella brasiliensis), foram as espécies destaque, em períodos distintos, apontadas como potenciais para produção de peixes marinhos no Brasil (Alvarez-Verde et al., 2015; Baldisserotto; Gomes, 2010; Baloi et al., 2014; 2017; Benetti et al., 2002; 2008; Benetti; Fagundes, 1980; Boglione et al., 2009; Bourque; Phelps, 2007; Boza-Abarca et al., 2008; Cabrera et al., 1998; Cabrita et al., 2009; Carvalho et al., 2010; 2019; Carvalho et al., 2019; Cavalli et al., 2011; Cavalli; Hamilton, 2009; Caylor et al., 1994; Cerqueira; Tsuzuki, 2009; Clarke et al., 1997; Cunha et al., 2013; Emata, 2003; Gesteira; Rocha, 1976; Glamuzina et al., 1998; Godinho et al.,1993; Guinle et al., 2015; Hamilton et al., 2013; Ibarra-Castro; Alvarez-Lajonchere, 2009; 2011; Kerber et al., 2012; Lanes et al., 2010; Leu et al., 2003; Liebl et al., 2016; Maltez et al., 2019; Marino et al., 2000; 2003; Muhlia-Melo et al., 2003; Okamoto et al., 2006; 2012; Papanikos et al., 2008; Passini et al., 2016; 2018; Pereira, 2010; Phelps et al., 2009; Rocha et al., 2008; Russo et al., 2009; Sampaio et al., 2007; 2016; Silva, 2013; Souza et al., 2016; Sterzelecki et al., 2017; Turano et al., 2000; Watanabe et al. 1998). Mas, apesar de todas as pesquisas e esforços concebidos no decorrer dos anos, a produção comercial da piscicultura marinha brasileira foi efetivada por um período exíguo, especificamente com a produção da espécie Rachycentron canadum.

De acordo com os pesquisadores entrevistados, inicialmente, em razão das perdas econômicas relacionadas à quebra de produção de camarões marinhos (Litopenaeus vannamei) por efeito de enfermidades, algumas empresas começaram a avaliar a oportunidade da produção de outras espécies. Foi neste contexto que o bijupirá (Rachycentron canadum), ou beijupirá ou cação de escama, despontou como espécie alvo nos estudos voltados para o desenvolvimento da piscicultura marinha no Brasil, por causa de suas características, em especial, o seu desempenho de crescimento em cativeiro, a viabilidade da produção de alevinos e pelo fato de ser uma espécie nativa.
A primeira desova da espécie ocorreu em 2006, no Laboratório de Reprodução de Peixes Marinhos, da empresa Bahia Pesca, podendo este ser considerado um marco na história da piscicultura marinha brasileira (Sampaio et al., 2010). Entretanto, foi a empresa Aqualíder que, no final de 2009, comercializou a primeira produção de bijupirá em cativeiro no Brasil, que correspondeu ao volume de 49 toneladas (MPA, 2011).

Foi por volta do ano de 2008 que a empresa Aqualíder começou a dedicar-se a produção de bijupirá em cativeiro, buscando, além da concessão de áreas, uma parceria com a Universidade Federal Rural de Pernambuco (UFRPE). De fato, a empresa adquiriu a permissão onerosa de uso, por 20 anos, de uma área total de 169 hectares, sendo 2,36 destinados especificamente ao cultivo, localizada $11 \mathrm{~km}$ da praia de Boa viagem, Pernambuco (Cavalli; Hamilton, 2009). Para a cooperação com a UFRPE, foi estabelecido e consolidado um protocolo brasileiro para a produção de alevinos. Então, a empresa implantou um laboratório especificamente para produção de alevinos de beijupirá e seguiu com a instalação de três gaiolas offshore (das 48 projetadas), onde ocorreu a primeira produção (engorda) brasileira em piscicultura marinha. Após este período, problemas como o dano de gaiolas por causa da colisão de embarcações, a má qualidade da ração disponível no mercado, o desenvolvimento de doenças, a inexistência no Brasil de seguro para atividade e a carência de mão de obra qualificada, ocasionaram o fechamento da empresa em 2010 (Cavalli et al., 2011).

No setor privado também se destacou a atuação da empresa Itapema, localizada em São Sebastião, São Paulo. Segundo um ex-empresário entrevistado, a Itapema iniciou sua produção de alevinos e engorda de Rachycentron canadum em gaiolas nearshore em 2011, mas em 2016, principalmente por motivos nutricionais e por questões de licenciamento ambiental, encerrou suas atividades.

No cenário do fomento público, em 2007, com o mesmo ímpeto de desenvolver tecnologias sustentáveis para criação do bijupirá, foi criada no Brasil a Rede de Pesquisa e Desenvolvimento em Piscicultura Marinha (REPIMAR), bem como duas sub-redes. A REPIMAR, com o projeto Desenvolvimento de tecnologias sustentáveis para a criação do beijupirá no Brasil, reuniu a expertise brasileira em piscicultura marinha para desenvolver estudos nos temas de genética, nutrição, sanidade, sistemas de produção e processamento do pescado em todo o território brasileiro. Esta rede foi composta por diversas instituições, como universidades públicas, fundações de pesquisa e a Embrapa e encerrou suas atividades em 2012.

Igualmente, em 2009, a UFRPE contou com o financiamento do então Ministério da Pesca e Aquicultura (MPA) para a execução do projeto Cação de Escama: cultivo de bei- 
jupirá pelos pescadores artesanais do litoral de Pernambuco, a partir do qual houve a instalação de uma fazenda de criação de beijupirá. Segundo o pesquisador responsável, o projeto tinha como objetivo capacitar pescadores da região metropolitana de Recife, determinar parâmetros técnicos e econômicos para viabilizar a criação sustentável do beijupirá nas condições brasileiras, além de colaborar com uma série de estudos científicos ligados à REPIMAR. O referido projeto encerrou suas atividades em dezembro de 2012.

Isso posto, pode-se pressupor que findaram as principais iniciativas, públicas e privadas, e o iminente vislumbre do desenvolvimento da cadeia produtiva da piscicultura marinha no Brasil que, atualmente, baseando-se nas pesquisas e entrevistas realizadas com os atuais e ex-integrantes deste setor, se resume a um laboratório privado de produção de alevinos de bijupirá e garoupa (Epinephelus marginatus), localizado em Ilha Bela - SP, e em quatro empresas privadas de engorda extensiva de bijupirá em gaiolas: uma na ilha de Búzios, São Sebastião - SP; uma em Ubatuba - SP; uma em Angra dos Reis - RJ; e uma em Vitória - ES. Também há uma parceria privada (Pousada Náutillus - RJ) e uma pública (Prefeitura do Rio de Janeiro e UERJ) para a produção de alevinos e engorda de bijupirá em gaiolas nearshore.

Duas empresas privadas comercializam rações específicas para espécies marinhas em granulometrias para as diferentes fases de desenvolvimento, umas das quais, segundo seu representante comercial, realiza a produção e a "batida" da ração, a partir da demanda mínima de $4.000 \mathrm{~kg}$ ou 160 sacas de $25 \mathrm{~kg}$ por milimetragem. Já os produtos de enriquecedores, básicos na produção de alimento vivo para larvicultura de peixes marinhos, segundo um ex-fornecedor, devido ao declínio da demanda, não estão mais disponíveis no mercado local. Segundo os empresários do setor de produção de alevinos, o que resta então é a importação desses produtos, que eleva o custo de produção, ou a elaboração de fórmulas artesanais que, segundo alguns técnicos dos laboratórios visitados, tem sido uma prática utilizada por alguns laboratórios de pesquisa atuantes no tema piscicultura marinha.

O fato é que a constatação realizada em 2003 por Roubach et al. (2003), de que a piscicultura marinha de corte não era uma atividade comercial no Brasil, continua válida. As informações disponíveis sobre a piscicultura marinha brasileira são basicamente oriundas de pesquisas científicas e, apesar de sua relevância, é uma das parcelas do empenho necessário para possibilitar o desenvolvimento da atividade. As circunstâncias apontam indícios de que as experiências passadas geraram receio sobre a viabilidade do setor e o recuo de investimentos, privados e públicos, tornando o cenário atual para o desenvolvimento da cadeia produtiva da piscicultura marinha no Brasil ainda mais desafiador. Desta forma, apesar do evidente potencial, a produção da piscicultura marinha em larga escala continua inexistente.

\section{Desafios e perspectiva da piscicultura marinha no estado do Ceará}

Segundo Ostrensky e Boeger (2008), a ausência de fornecedores de alevinos em escala comercial, de oferta de rações comerciais adequadas, da determinação de áreas para cultivos, de infraestrutura de apoio ao mercado e a dificuldade de licenciamento ambiental são os principais desafios para o estabelecimento da cadeia produtiva de peixes marinhos. A falta de oferta local dos demais insumos (enriquecedores de alimento vivo), adquiridos via importação e da mão de obra qualificada são outros fatores que igualmente devem ser considerados, visto a contribuição significativa destes fatores no incremento dos custos de produção. Portanto, por ser uma atividade de custo elevado e potencial de aumento de produção em longo prazo (Ostrensky et al., 2008), o investimento de recursos no setor deve ser preconizado e ponderado por estudos de viabilidade econômica e de execução.

Até o presente momento não há registros de produção de piscicultura marinha para corte (produção de carne) no estado do Ceará, sendo a pesca a grande responsável por suprir esta demanda. Entretanto, igualmente ao cenário mundial e brasileiro, os estoques pesqueiros cearenses de interesse comercial estão na lista de espécies ameaçadas de sobre-explotação ou sobre-explotadas (MMA, 2005), reiterando a necessidade de alternativas eficazes e sustentáveis para promover o fornecimento de pescado de origem marinha (Camargo; Pouey, 2005; Gonçalves, 2011). Ciente que a pesca é cada vez mais ineficaz em atender a demanda crescente, o governo do estado do Ceará busca identificar essas alternativas.

O potencial do Ceará para o desenvolvimento da piscicultura marinha é notório. O estado possui $573 \mathrm{~km}$ de litoral com características que possibilitam o desenvolvimento da piscicultura marinha, além da ausência de oscilações significativas na temperatura e de possuir em sua fauna nativa espécies apreciadas pelos consumidores de valor de mercado, como os "peixes vermelhos". O termo "peixes vermelhos" se refere àqueles pertencentes à família Lutjanidae, detentora de destaque no nível mundial, nacional e regional (Popma; Masser, 1999; Benetti et al., 2002; Velarde et al., 2012), sendo usualmente comercializados na forma "inteiro", "fresco" ou "congelado", com preço médio no Ceará variando, de acordo com a espécie, entre 25,00 e 14,00 reais por quilo (Allen, 1985; Ceasa-CE, 2020).

O Ceará desponta neste cenário, contemplando ao seu Plano de Desenvolvimento da Aquicultura do Estado do Ceará ações voltadas para promover o desenvolvimento sustentável da piscicultura marinha no estado. Estas medidas têm sido promovidas e executadas através da Fundação Cearense de Apoio ao Desenvolvimento Científico e Tecnológico (FUNCAP), por meio do Programa Cientista Chefe, associado 
à Coordenadoria de Desenvolvimento da Pesca e Aquicultura Familiar (COPEA) da Secretaria do Desenvolvimento Agrário (SDA). Segundo o cientista chefe do núcleo aquicultura do programa, as ações estão incialmente direcionadas ao fornecimento de alevinos de espécies de peixes marinhos e a análise das espécies aptas para este fim. Como medida para o fornecimento de alevinos, está sendo elaborado um projeto de laboratório para a produção alevinos de peixes marinhos em conjunto à execução de um estudo de viabilidade econômica e financeira.

Já as ações de prospecção de espécies aptas para produção em cativeiro no estado do Ceará têm verificado que, dentre as espécies de peixes marinhos apontados como propícias à produção em cativeiro, estão dois Lutjanídeos: Lutjanus synagris e Lutjanus analis (Watanabe et al., 1998; Benetti et al., 2002; Botero; Ospina, 2002; Vettorazzi et al., 2010; Cerqueira et al., 2017). Porventura, a produção comercial de Lutjanídeos já é uma realidade em vários países, como Costa Rica (L. guttatus), Hon Kong ( $L$. russelli), Taiwan (L. bohar), Singapura (L. goldiei), Filipinas (L. spp.) e Malásia (L. argentimaculatus) (Lucas; Southgate, 2012). Oportunamente, Lutjanus synagris e Lutjanus analis fazem parte do grupo de espécies nativas destacadas anteriormente pelo apreço por parte dos consumidores e por seu preço de mercado.

A espécie Lutjanus synagris (ariacó) distribui-se no Oceano Atlântico Ocidental, desde o estado da Carolina do Norte (EUA) até o estado de São Paulo (Brasil) (Souza et al., 2016). Podem atingir até $60 \mathrm{~cm}$, apresentando maturidade sexual entre 15 a $18 \mathrm{~cm}$ e podendo chegar a 3,8 kg (Sanches; Cerqueira, 2010). Já o Lutjanus analis (cioba), considerada uma das espécies de peixes marinhas mais saborosas (Watanabe et al., 1998), ocorre no Atlântico Ocidental, de Nova Inglaterra até o sudeste do Brasil, mas que tem apresentando uma drástica redução na sua abundância e distribuição (Menezes; Figueiredo, 1980; Anderson, 2002; Menezes et al., 2003; Ávila-da-Silva et al., 2007; Froese; Pauly, 2008). Podem atingir $80 \mathrm{~cm}$ e pesar $11 \mathrm{~kg}$, alcançado a maturidade sexual aos quatro anos, quando apresentam comprimento superior a $50 \mathrm{~cm}$ (Claro; Lindeman, 2008). Ambas as espécies possuem hábito alimentar carnívoro e alimentam-se principalmente de peixes e crustáceos (Randall, 1967; Menezes; Figueiredo, 1980; Bohlke; Chaplin, 1993; Anderson, 2002; Menezes et al., 2003; Ávila-da-Silva et al., 2007; Froese; Pauly, 2008).

As ações de prospecção de espécies verificaram que vários estudos no Brasil e no Ceará constataram que Lutjanus synagris e Lutjanus analis se adaptam ao cativeiro e ao consumo de alimento inerte (ração), são espécies rústicas, resistentes ao manejo e com potencial de crescimento em cativeiro (Watanabe et al., 1998; Benetti et al., 2002; Botero; Ospina, 2002; Vettorazzi et al., 2010, Freitas et al., 2011). Entretanto, se observa que, enquanto a bibliografia relacio- nada ao ariacó (Lutjanus synagris) apresenta dados referentes à sua desova em cativeiro e registro de larvicultura pontualmente até o $30^{\circ}$ dia após a eclosão (Facundo, 2016; Souza, 2012; Souza et al., 2016), os dados sobre a cioba ( $L$. analis) retratam desovas, larvicultura e produção de juvenis bem-sucedidas, além de apontá-la como umas das melhores perspectivas para produção comercial (Clarke et al., 1997; Watanabe et al., 1998; Feeley; Benetti, 1999; Feeley et al., 2000; Watanabe et al., 2001; Benetti et al., 2002; Botero-Arango; Castano-Rivera, 2005). À vista disso, a prospecção de espécies tem se voltado especialmente para Lutjanus synagris (ariacó) e, sobretudo, Lutjanus analis (cioba).

Mediante a continuidade das iniciativas e ações de fomento, pesquisas, bem como de investimentos públicos e privados, o cenário aponta uma possibilidade promissora para a produção comercial de piscicultura marinha para corte no estado do Ceará.

\section{CONCLUSÃO}

No decorrer dos anos, pesquisas científicas foram realizadas, inciativas públicas e privadas foram efetivadas e, em menor escala, empreendimentos foram implementados. Entretanto, o desenvolvimento da piscicultura marinha no Brasil segue incipiente e com obstáculos a serem superados. Apesar das experiências passadas e dos pormenores que dificultam e entravam a sua evolução no Brasil, a piscicultura marinha é uma realidade em outros países e continua sendo mundialmente apontada como opção viável, principalmente no que se refere à manutenção do fornecimento de pescado como fonte de proteína de alta qualidade, mediante o crescimento populacional; à redução da exploração dos estoques pesqueiros comerciais; e ao desenvolvimento regional. Esses propósitos são fundamentos para persistir no aprimoramento e na busca de inovações e estratégias para impulsionar o setor e finalmente converter a circunstância brasileira de potencial para efetiva.

No que concerne ao Ceará, algumas ações para promoção da piscicultura marinha têm sido executadas. Os resultados parciais dessas medidas proveram a elaboração de um projeto de laboratório para fornecer alevinos de espécies marinhas para o estado, em conjunto à execução de um estudo da viabilidade econômica e financeira, e a prospecção de espécies para produção em cativeiro têm se voltado preliminarmente para Lutjanus synagris (ariacó) e, sobretudo, Lutjanus analis (cioba).

\section{REFERÊNCIAS}

Allen, G.R. (1985). An annotated and illustrated catalogue of lutjanid species known to date. FAO Species Catalogue, Vol. 6, Snappers of the world. Rome: FAO, disponível em: http:// 
www.fao.org/3/ac481e/ac481e00.htm (acesso em 10 maio 2020).

Alvarez-Verde, C.A.; Sampaio, L.A.; Okamoto, M.H. (2015), "Effects of light intensity on growth of juvenile Brazilian flounder Paralichthys orbignyanus", Boletim do Instituto de Pesca, Vol. 41, pp. 859-864, disponível em: https://www. pesca.sp.gov.br/boletim/index.php/bip/article/view/1078 (acesso em 11 de maio 2020).

Anderson, W.D. (2002), "Lutjanidae”, in Carpenter K. E. (ed.), The living marine resources of the Western Central Atlantic. Volume 3: Bony fishes part 2 (Opistognathidae to Molidae), sea turtles and marine mammals. FAO Species Identification Guide for Fishery Purposes and American Society of Ichthyologists and Herpetologists Special Publication № 5. Rome: FAO. p. 1479-1504.

Ávila-da-Silva, A.O.; Carneiro, M.C.; Mendonça, J.T.; Servo, G.J.M.; Bastos, G.C.C.; Batista, P.A. (2007), "Produção pesqueira marinha do estado de São Paulo no ano 2005", Série Relatórios Técnicos do Instituto de Pesca, São Paulo, Vol. 26, pp. 1-44.

Baldisserotto, B.; Gomes, L.C. (2010), “Espécies nativas para a piscicultura no Brasil", 2 ed., Santa Maria, UFSM.

Baloi, M.; Carvalho, C.V.A.; Sterzelechi, F.; Passini, G.; Cerqueira, V. (2014), "Effects of feeding frequency on growth, feed effciency and body composition of juveniles Brazilian sardine, Sardinella brasiliensis (Steindacher 1879)", Aquaculture Research, Vol. 47, No. 2, pp.554-560.

Baloi, M.F., Sterzelecki, F.C., Sugai, J.K., Passini, G., Carvalho, C.V.A.; Cerqueira, V.R. (2017), "Growth performance, body composition and metabolic response to feeding rates in juvenile Brazilian sardine Sardinella brasiliensis", Aquaculture Nutrition, Vol. 23, No. 6, pp.1458-1466.

Benetti, D.D.; Fagundes Netto, E.B. (1980), “Considerações sobre desova e alevinagem da tainha (Mugil Liza Valenciennes, 1836)", Instituto de Pesquisas da Marinha, Rio de Janeiro, Brasil, Vol. 135, pp. 1-26.

Benetti, D.D.; Matera, J.A.; Stevens, O.M.; Alarcón, J.F.; Feeley, M.W.; Rotman, F.J.; Minemoto, Y.; Banner-Stevens, G.; Fanke, J.; Zimmerman, S.; Eldridge, L. (2002), "Growth, Survival and Feed Conversion Rates of Hatchery-reared Mutton Snapper Lutjanus analis Cultured in Floating Net Cages", Journal of the World Aquaculture Society, Vol. 33, No. 3, pp. 349-357.

Benetti, D.D.; Sardenberg, B.; Welch, A.; Hoenig, R.; Orhun, M.R; Zink I. (2008), "Intensive larval husbandry and fingerling production of cobia Rachycentron canadum", Aquaculture, Vol. 281, pp. 22-27.

Boglione, C.; Marino, G.; Giganti, M.; Longobardi, A.; De Marzi, P.; Cataudella, S. (2009), "Skeletal anomalies in dusky grouper Epinephelus marginatus (Lowe 1834) juveniles reared with different methodologies and larval densities", Aquaculture, Vol. 291, No.1-2, pp. 48-60.

Bohlke, J.E.; Chaplin, C.G. (1993), "Fishes of the Bahamas and adjacente tropical waters", $2^{\text {nd }}$ edition, Austin, University of Texas, Press. 771p.

Botero, J.; Ospina, J.F. (2002), “Crecimiento de juveniles del pargo palmero Lutjanus analis (Curvier) em jaulas flotantes en islas del Rosario, Caribe Colombiano", Bol. Invest. Mar. Cost., Vol.31, p.205-217.

Botero-Arango, J.; Castano-Rivera, F. (2005), "Inducción de la madurez gonadal del pargo palmero Lutjanus analis (Pisces: Lutjanidae) mediante la aplicación de un fototermoperiodo artificial de acondicionamento", Bol. Invest. Mar. Cost., Vol.34, p.69-79.

Bourque, B.D.; Phelps, R.P. (2007), “Induced spawning and egg quality evaluation of red snapper, Lutjanus campechanus", Journal of the World Aquaculture Society, Vol. 38, No. 2, pp. 208-217.

Boza-Abarca, J.; Calvo-Vargas, E.; Solis-Ortiz, N.; Komen, H. (2008), "Induced spawning and larval rearing of spotted rose snapper, Lutjanus guttatus, at the Marine Biology Station, Puntarenas, Costa Rica", Ciencias Marinas, North America, Vol. 34, No. 2, pp. 239-252.

Brasil. (2009). Lei n 11.959, 29 de junho de 2009. Diário Oficial da União, 30 jun. 2009.

Cabrera, J.R.; Barrios, T.C.; Quijada, J.M. (1998), “Inducción al desove del pargo de mangle, Lutjanus griseus LINNAEUS (Pisces: Lutjanidae), sexualmente maduro en cautiverio", Arquivos de Ciências do Mar, Vol. 31, pp. 57-63.

Cabrita, E.; Engrola, S.; Conceição, L.E.C.; Pousão-Ferreira, P.; Dinis, M.T. (2009), "Successful cryopreservation of sperm from sex-reversed dusky grouper, Epinephelus marginatus, Aquaculture, Vol. 287, No. 1-2, pp. 152-157.

Camargo, S.G.O.; Pouey, J.L.O.F. (2005), "Aquicultura - um mercado em expansão", Revista Brasileira de Agrociência, Vol. 11, No. 4, pp. 393-396.

Carvalho, C.V.A.; Bianchini, A. ; Tesser, M.B.; Sampaio, L.A. (2010), "The effect of protein levels on growth, postprandial excretion and tryptic activity of juvenile mullet Mugil platanus (Günther)", Aquaculture Research (Print), Vol. 41, pp. 511-518.

Carvalho, C.V.A.; Passini, G.; Sterzelecki, F.C.; Baloi, F.M.; Cerqueira, V.R. (2019), "Maturation, spawning and larviculture of the mullet Mugil liza under laboratory conditions", Revista Brasileira de Reprodução Animal, jan./mar.,Vol. 43, No. 1, pp. 31-36.

Cavalli, R.O.; Domingues, E.C.; Hamilton, S. (2011), "Desenvolvimento da produção de peixes em mar aberto no Brasil: possibilidades e desafios", Revista Brasileira de Zootecnia, Vol. 40, pp. 155-164. 
Cavalli, R.O.; Hamilton, S. (2007), "A piscicultura marinha no Brasil. Afinal, quais as espécies boas para cultivar", Panorama da Aquicultura, Vol. 17, No. 104, nov-dez.

Cavalli, R.O.; Hamilton, S. (2009), "Piscicultura marinha no Brasil com ênfase na produção do beijupirá", Rev. Bras. Reprod. Anim. Supl., Vol. 6, pp.64-69.

Caylor, R.E.; Biesiot, P.M.; Franks, J.S. (1994), "Culture of cobia Rachycentron canadum: cryopreservation of sperm and induced spawning", Aquaculture, Vol.125, pp. 81-92.

Ceasa - CE (2020). Centrais do Abastecimento do Ceará S/A. Sistema de Informação de Mercado Agrícola-SIMA/CE, disponível em: https://files.ceasa-ce.com.br/unsima/comparativo_precos/comparativo.html (acesso em 21 de julho 2020).

Cerqueira, V.C.; Carvalho, C.V.A.; Sanches, E.G.; Passini, G.; Baloi, M.F.; Rodrigues, R.V.R. (2017), “Manejo de reprodutores e controle da reprodução de peixes marinhos da costa brasileira", Ver. Bras. Reprod. Anim., Belo Horizonte, Vol. 41, No. 1, jan./mar., pp.94-102.

Cerqueira, V.R. (2004), "Cultivo de Peixes Marinhos", in Poli C. R.; Poli A.T.; Andreatta E.; Beltrame E. (ORG.). Aquicultura: Experiências Brasileiras, Florianópolis, Multitarefa editora Ltda, Vol. 1, pp. 369-406.

Cerqueira, V.R; Tsuzuki, M.Y. (2009), “A review of spawning induction, larviculture, and juvenile rearing of the fat snook, Centropomus parallelus", Fish Physiol Biochem, Vol. 35, pp. 17-28.

Clarke, M.E.; Domeier, M.L.; Laroche, W.A. (1997), “Development of larvae and juveniles of the mutton snapper (Lutjanus analis), lane snapper (Lutjanus synagris) and yellowtail snapper (Lutjanus chrysurus)", Bulletin of Marine Science, Vol. 61 No. 3, pp. 511-537.

Claro, R.; Linderman, K.C. (2008), "Biología y manejo de los pargos (Lutjanidae) en el Atlántico occidental”, La Habana: Instituto de Oceanología, 2008. 472p.

Côrtes, G.F.; Tsuzuki, M.Y. (2010), "Efeito do tamanho do rotífero na sobrevivência e no crescimento de neon gobi Elacatinus fígaro durante as fases iniciais de larvicultura", Boletim do Instituto de Pesca, Vol. 36, No. 3, pp. 205-212.

Cunha, M.E.; Quental-Ferreira, H.; Gavaia, P. J.; Pousao-Ferreira, P. (2013), "Larval and juvenile development of dusky grouper Epinephelus marginatus reared in mesocosms", J. Fish. Biol., Vol. 83, p. 448-465.

Cunha, V.L.; Shei, M.R.P.; Okamoto, M.H.; Rodrigues, R.V.; Sampaio L.A. (2013), "Feeding rate and frequency on juvenile pompano growth", Pesq. Agrop. Bras., Brasília, Vol. 48, No. 8, pp. 950-954.

Emata, A.C. (2003), "Reproductive performance in induced and spontaneous spawning of the mangrove red snapper
Lutjanus argentimaculatus: A potential candidate species for sustainable aquaculture", Aquaculture Research, Vol. 34, pp. 849-857.

Facundo, G.M. (2016), Captura, aclimatação e manejo de reprodutores de Lutjanídeos, Lutjanus sp., e a indução à reprodução em cativeiro do ariacó $L$. synagris", Dissertação de mestrado em Ciências Marinha Tropical, Universidade Federal do Ceará, Fortaleza, CE.

Feeley, M.; Benetti, D.D. (1999), "Spawning and larval husbandry of mutton snapper, Lutjanus analis, mangrove snapper, L. griseus, and yellowtail snapper, Ocyurus chrysurus, three tropical lutjanid species", artigo apresentado no World Aquaculture '99, Annual International Conference and Exposition of the World Aquaculture Society, 26 April-2 May 1999, Sydney, Australia.

Feeley, M.W.; Benetti, D.D.; Stevens, O.; Famke, J.; Alarcon, J.; Matera; Stevens, G.; Eldrige, L. (2000), "Spawning, larval rearing and fingerling production of mutton snapper", World Aquaculture Society. U.S. Chapter. Book of Abstracts, Aqua America 2000, New Orleans, Louisiana.

Food and Agriculture Organization of the United States - FAO (2020), "The State of World Fisheries and Aquaculture 2020 - Sustainability in Action", Rome, disponível em: http:// www.fao.org/3/ca9229en/CA9229EN.pdf (acesso em 8 de junho 2020)

Freitas, E.L.; Nunes, A.J.P, Sá, M.V.C. (2011), “Growth and feeding responsesof mutton snapper, Lutjanus analis (Cuvier 1828), fed on diets with soy protein concentrate in replacement of Anchovy fish meal", Aquaculture Research, Vol. 42, pp. 866-877, disponível em: https://onlinelibrary.wiley.com/ doi/epdf/10.1111/j.1365-2109.2010.02793.x (acesso em 17 de junho 2020).

Froese, R.; Pauly, D. (2008), “Fishbase”, Disponível em: www. fishbase.org (acesso em 11 de meio 2020).

Gesteira, T.C.V.; Rocha, C.A.S. (1976), "Estudo sobre a fecundidade do ariacó, Lutjanus synagris (Linnaeus), da costa do Estado do Ceará (Brasil)", Arquivos de Ciência do Mar, Vol. 16, No. 1, pp. 19-22.

Glamuzina, B.; Glavic, N.; Skaramuca, B.; Kozul, V. (1998), "Induced sex reversal of dusky grouper, Epinephelus marginatus (Lowe)", Aquaculture Research, Vol. 29, No. 8, pp. 563-567.

Godinho, H.M.; Kavamoto, E.T.; Andrade-Talmelli, E.F.; Serralheiro, P.C.S.; Paiva, P.; Ferraz, E.M. (1993), "Induced spawning of the mullet Mugil platanus GUNTHER, 1880, in Cananéia, São Paulo, Brazil", Bol. Inst. Pesca, Vol.20, pp.5966.

Gonçalves, A.A. (Org.) (2011), "Tecnologia do pescado: ciência, tecnologia, inovação e legislação", São Paulo, Editora Atheneu, 593pp. 
Guinle, L.V.; Passini, G.; Carvalho, C.V.A.; Cerqueira, V.R. (2015), "Viabilidade econômica da produção de juvenis de robalo-flecha (Centropomus undecimalis), Estado de Santa Catarina", Informações Econômicas (Online), Vol. 45, pp. 49, disponível em: http://www.iea.sp.gov.br/ftpiea/publicacoes/ie/2015/tec05-0615.pdf (acesso em 11 de maio 2020).

Hamilton, S.; Deveri, W.; Cavalli, R.O. (2013), "Biologia e aquicultura do beijupirá: uma revisão", Bol. Inst. Pesca, São Paulo, Vol. 39, No. 4, pp. 461-477.

Hirata, H. (1979), "Rotifer culture in Japan", in Stycznska-Juurewicz T.;Backiel E., Cultivation of fish fry ans its live food, European Mariculture Society, Special Publication, Bredene, Vol. 4, pp. 361-375.

Ibarra-Castro, L.; Alavarez-Lajonchere, L. (2009), “Improved Induced-Spawning Protocol for the Spotted Rose Snapper (Lutjanus guttatus)", The Israeli Journal of Aquaculture, Vol. 61, No. 2, pp. 121-133.

Ibarra-Castro, L.; Alvarez-Lajonchere, L. (2011), "GnRHa-induced Multiple Spawns and Volition Spawning of Captive Spotted Rose Snapper, Lutjanus guttatus, at Mazatlan, Mexico", Journal of the World Aquaculture Society, Vol. 42, No. 4, p. 564-574.

Kerber, C.E.; Azevedo, Silva, H.K.; Santos, P.A.; Sanches, E.G. (2012), "Reproduction and larviculture of dusky grouper Epinephelus marginatus (Lowe 1834) in Brazil", J. Agricult. Sci. Technol., Vol.2, pp.229-234.

Lanes, C.F.C.; Okamoto, M.H.; Bianchini, A.; Marins, L.F.; Sampaio, L.A. (2010), "Sperm quality of Brazilian flounder Paralichthys orbignyanus throughout the reproductive season", Aquaculture Research, Vol. 41, pp. 199-207.

Leu, M.Y.; Fang, L.S.; Chen, I.H. (2003), “Natural spawning and rearing of mangrove red snapper, Lutjanus argentimaculatus, larvae in captivity", The Israeli Journal of Aquaculture, Vol. 55, No. 1, pp. 22-30.

Liebl, F.; Amaral, Jr. H.; Garcia, S.; Souto, L.; Carvalho, C.V.A.; Cerqueira, V.R. (2016), "Desempenho de juvenis de robalo-flecha e robalo-peva submetidos a diferentes densidades de estocagem em água doce", Boletim do Instituto de Pesca (Online), Vol. 42, pp. 145-155, disponível em: https://www.pesca.sp.gov.br/DOI2016/sumario42_1/12BIP1202artigo145-155WEB.htm (acesso em 11 de maio 2020).

Lucas, J.S.; Southgate, P.C. (2012), "Aquaculture: Farming Aquatic Animals and Plants", Wiley-Blackwell, $2^{\circ}$ Edição, 6 de janeiro de 2012, 648pp.

Maltez, L.C.; Barbas, L.A.; Okamoto, M.H; Alcantara, D.L.; Romano, L.A.; Sampaio, L.A.; Garcia, L. (2019), "Secondary Stress Responses in Juvenile Brazilian Flounder, Paralichthys orbignyanus, throughout and after Exposure to Sublethal Levels of Ammonia and Nitrite", Journal of the World Aquaculture Society, Vol. 50, pp. 346-358.
Marino G.; Panini, E.; Longobardi, A.; Mandich, A.; Finoia, M.; Zohar, Y.; Mylonas, C.C. (2003), "Induction of ovulation in captive-reared dusky grouper, Epinephelus marginatus (Lowe, 1834), with a sustained-release GnRHa implant", Aquaculture, Vol. 219, No. 1-4, pp. 841-858.

Marino, G.; Azzurro, E.; Finoia, M.G.; Messina, M.C.; Massari, A.; Mandich, A.; (2000), "Recent advances in induced breeding of the dusky grouper Epinephelus marginatus (Lowe, 1834), in Chioccioli, E. (Ed.), Recent Advances in Mediterranean Aquaculture Finfish Species Diversification, Proceedings of the Seminar of the CIHEAM Network on Technology of Aquaculture in the Mediterranean (TECAM), jointly organized by CIHEAM and FAO, 24 May- 28 May 1999, Zaragoza, Spain. Cah. OptionsMediterr., Vol. 47, CIHEAM/ FAO, pp. 215- 225.

Menezes, N.A.; Figueiredo, J.L. (1980), Manual de peixes marinhos do sudeste do Brasil. IV. Teleostei (3), São Paulo: Museu de Zoologia da USP. 96p.

Menezes, N.A.M.; Buckup, P.A.; Figueiredo, J.L.; Moura, R.L. (Eds) (2003), "Catálogo das espécies de peixes marinhos do Brasil", São Paulo: Museu de Zoologia da USP. 159p.

Ministério da Pesca e Aquicultura (MPA) (2011), "Boletim estatístico da pesca e aquicultura Brasil 2008-2009", Brasília: MPA, $100 \mathrm{p}$.

Ministério do Meio Ambiente (MMA) (2005), "Instrução normativa n.o 52, de 8 de novembro de 2005. Altera os Anexos I e II da Instrução Normativa $\mathrm{n}^{\circ} 5$ do Ministério do Meio Ambiente, de 21 de maio de 2004", publicada no Diário Oficial da União de 28 de maio de 2004, Seção 1, p.136-142.

Muhlia-Melo, A.; Guerrero-Tortolero, D.A.; Perez-Urbiola, J.C.; Campos-Ramos, R. (2003), "Results of spontaneous spawning of yellow snapper (Lutjanus argentiventris Peters, 1869) reared in inland ponds in La Paz, Baja California Sur, México", Fish Physiology and Biochemistry, Vol. 28, pp. 511-512.

Okamoto, M.H.; Sampaio, L.A. (2012), "Sobrevivência e crescimento de juvenis do linguado Paralichthys orbignyanus criados em deferentes temperaturas, Atlântica, Vol. 34, pp. 57-61.

Okamoto, M.H.; Sampaio, L.A.; Maçada, A.P. (2006), “Efeito da temperatura sobre o crescimento e a sobrevivência de juvenis da tainha Mugil platanus GÜNTHER, 1880", Atlântica, Vol. 28, No.1, pp. 61-66.

Ostrensky A.; Boeger W. A.; Chammas M. A. (2008), "Potencial para o desenvolvimento da aquicultura no Brasil", in Ostrensky, A.; Borghetti, J.R.; Soto, D., Aquicultura no Brasil: o desafio é crescer, Brasília, SEAP, pp. 159-182.

Ostrensky, A.; Boeger, W.A. (2008), "Principais problemas enfrentados atualmente pela aquicultura brasileira", in Ostrensky, A.; Borghetti, J.R.; Soto, D., Aquicultura no Brasil: o desafio é crescer, Brasília, SEAP, p.135-158. 
Papanikos, N.; Phelps, R.P.; Davis, D.A.; Ferry, A.; Maus, D. (2008), "Spontaneous spawning of captive red snapper, Lutjanus campechanus, and dietary lipid effect on reproductive performance", Journal of the World Aquaculture Society, Vol. 39, No. 3, pp. 324-338.

Passini, G.; Carvalho, C.V.A.; Sterzelechi, F.C.; Cerqueira, V.R. (2016), "Induction of sex inversion in common snook (Centropomus undecimalis) males, using 17- $\beta$ oestradiol implants", Aquaculture Research (Print), Vol. 47, pp. 10901099.

Passini, G.; Sterzelecki, F.C.; Carvalho, C.V.A.; Baloi, M.F.; Naide, V.; Cerqueira, V.R. (2018), "17 $\alpha$-Methyltestosterone implants accelerate spermatogenesis in common snook, Centropomus undecimalis, during first sexual maturation", Theriogenogy, Vol. 106, pp. 134-140.

Pereira, H. L. (2010), “Manejo e maturação em cativeiro da sardinha-verdadeira Sardinella brasiliensis (Steindachner, 1879) no sul do Brasil, Dissertação de Mestrado em Aquicultura, Universidade Federal de Santa Catarina, Florianópolis, RS.

Phelps, R.P.; Papanikos, N.; Bourque, B.D.; Bueno, F.T.; Hastey, R.P.; Maus, D.L.; Ferry, A.; Davis, D.A. (2009), "Spawning of red snapper (Lutjanus campechanus) in response to hormonal induction or environmental control in a hatchery setting", Reviews in Fisheries Science, Vol. 17, No. 2, pp.149155.

Pillay, T.V.R. (1993), “Aquaculture. Principles and Practices”, Fishing News Book. 575pp.

Popma, T.; Masser, M. (1999), "Tilapia - Life history and biology", Southern Regional Aquaculture Cebter - SRAC, Auburn: Special Publication, No. 283, disponível em: https:// srac.tamu.edu/fact-sheets/search (acesso em 4 maio 2020).

Randall, J.E. (1967), "Food habits of reef fishes of the West Indies", Studies of Tropical Oceanography, Vol.5, pp.665-847.

Rocha, A.F.; Carvalho, C.V.A.; Sampaio, L.A. (2008), "Produção de juvenis do linguado Paralichthys orbignyanus: efeito da duração do período de co-alimentação durante o desmame”, Ciência Rural, Vol. 38, pp. 2334-2338.

Roubach, R.; Correia, E.S.; Zaiden, S.; Martino, R.C.; Cavalli, R.O. (2003), "Aquaculture in Brazil", World Aquaculture, Vol. 34 , pp. 25-35.

Russo, T.; Boglione, C.; De Marzi, P.; Cataudella, S. (2009), "Feeding preferences of the dusky grouper (Epinephelus marginatus, Lowe 1834) larvae reared in semi-intensive conditions: A contribution addressing the domestication of this species", Aquaculture, Vol. 289, No. 3-4, pp. 289-296.

Sampaio, L.A.; Ferreira, A.H. ; Tesser, B.M. (2001), "Effect of stocking density on laboratory rearing of mullet fingerlings, Mugil platanus (Gunther, 1880)", Acta Scientiarum (UEM), Maringá, Vol. 23, No. 2, pp. 471-475.
Sampaio, L.A.; Freitas, L.S.; Okamoto, M.H.; Louzada, L.R.; Rodrigues, R.V.; Robaldo, R.B. (2007), "Effects of salinity on Brazilian flounder Paralichthys orbignyanus from fertilization to juvenile settlement", Aquaculture (Amsterdam), Vol. 262, pp. 340-346.

Sampaio, L.A.; Okamoto, M.H.; Rodrigues, R.V.; Tesser, M.B. (2016), "Piscicultura marinha: Criação de bijupirá em sistema de recirculação de água", ed. 1, Rio Grande, Brasil, Editora da FURG, pp. 1-124.

Sampaio, L.A.; Tesser, M.B.; Wasielesky Jr. (2010), “Avanços da maricultura na primeira década do século XXI: piscicultura e carcinocultura marinha", Revista Brasileira de Zootecnia, Vol. 39, pp. 102-111.

Sanches, E.G.; Cerqueira, V.R. (2010), "Refrigeração do sêmen do ariacó Lutjanus synagris", Bol. Inst. Pesca, São Paulo, Vol. 36, No. 4, pp.293-305, disponível em: https://www.pesca.sp.gov.br/boletim/index.php/bip/article/view/925/906 (acesso em 12 de maio 2020).

Schubart, O. (1936), "Investigações sobre os viveiros do Recife", Bol. Sec. Agric. Ind. e Com. do Estado de Pernambuco, Vol. 1, No. 2, pp. 153-176.

Silva, C. A. (2010), "Rede de piscicultura marinha busca tecnologias para criação do bijupirá no Brasil", Beijupirá News, Ano 1, No. 3, pp.4, disponível em: https://labomar.ufc.br/ wp-content/uploads/2017/01/beijupira-news-ano1-no-3. pdf (acesso em 11 de maio 2020).

Silva, L.A.R. (2013), “Crescimento de juvenis, maturação sexual, reprodução e larvicultura da sardinha-verdadeira (Sardinella brasiliensis) em cativeiro", Dissertação de Mestrado em Aquicultura, Universidade Federal de Santa Catarina, Florianópolis, RS.

Souza, R.L.M. (2012), Reprodução induzida do ariacó, Lutjanus synagris (LINNAEUS, 1758), em cativeiro, Tese de doutorado em Ciências Marinhas Tropicais, Universidade Federal do Ceará, Fortaleza, CE.

Souza, R.L.M.; Vettorazzi, M.B.; Kobayashi, R.K.; Furtado Neto, M.A.A. (2016), Reprodução do Ariacó, Lutjanus synagris (Linnaeus, 1758), Sob Cultivo, em Resposta a Indução Hormonal", Arq. Ciên. Mar, Fortaleza, Vol. 49, No. 2, pp. 6876.

Sterzelecki, F.C.; Sugai, J.K.; Baloi M.; Passini, G.; Carvalho, C.V.A.; Fracalossi, D. M.; Cerqueira, V.R. (2017), "Effects of increasing protein level on the performance, enzyme activity and body composition of the Brazilian sardine, Sardinella brasiliensis (Steindachner, 1879)", Aquaculture Nutrition, Vol. 1, pp. 1-9.

Turano, M. J.; Davis, D.A.; Arnold, C.R. (2000), “Observations and techniques for maturations, spawning and larval rearing of the yellow tail snapper Ocyurus chrysurus", Journal of the World Aquaculture Society, Vol. 31, No. 1, pp. 59-68. 
Tutman, P.; Glavic, N.; Kozul, V.; Skaramuca, B.; Glamuzina, B. (2004), "Preliminary information on feeding and growth of pompano, Trachinotus ovatus (Linnaeus 1758) (Pisces; Carangidae) in captivity", Aquaculture International, Vol.12, pp.387-393.

Velarde, D.; Lara, C.; Durán M.; Bartlett, F.; Sardenberg, B.; Benetti, D. (2012), "Integrated sopotted red snapper aquaculture in Central America", Global Aquaculture Advocate, data, disponível em: https://www.aquaculturealliance.org/ advocate/integrated-spotted-red-snapper-aquaculture-central-america/ (acesso em 7 maio de 2020).

Vettorazzi, M.B.; Teixeira, E.G.; Souza, R.L.M.; César, J.R.O.; Furtado Neto, M.A.A. (2010), "Motilidade espermática do sêmen do Ariacó, Lutjanus synagris", Arquivos de Ciências do Mar, Vol. 43, No. 2, pp. 21-26.
Von Ihering R. (1932), "Criação de peixes em viveiros no Recife", Bol. Sec. Agric. Ind. Viação - Recife - PE, Vol.35, pp. 35-40.

Watanabe, W.; Benetti, D.; Feeley, M.; Davis D. A.; Phelps R. (2001), "Status of artificial propagation of mutton, yellowtail and red snapper (family Lutjanidae) in the southeastern U.S.", artigo apresentando no World Aquaculture Society Conference 2001, Orlando, Florida, 15-25 January 2001.

Watanabe, W.O.; Ellis, E.P.; Ellis, S.C.; Chaves, J.; Manfredi, C.; Hagood, R.W.; Sparsis, M.; Arneson, S. (1998), "Artificial Propagation of Mutton Snapper, Lutjanus analis, a New Candidate Marine Fish Species for Aquaculture". Journal of the World Aquaculture Society, Vol. 29, No. 2, pp. 176-187.

Recebido: 14 maio. 2020

Aprovado: 30 jun. 2020

DOI: 10.20985/1980-5160.2020.v15n2.1636

Como citar: Lisboa, V., Eloy, H.R.F., Catter, K.M., et al. (2020). Piscicultura marinha brasileira: desafios e perspectivas do seu desenvolvimento no estado do Ceará. Revista S\&G 15, 2, 113-122. https://revistasg.emnuvens.com.br/sg/ article/view/1636 